\title{
Innovación social y participación efectiva de niños, niñas y adolescentes
}

\author{
David Vila Viñas ${ }^{1}$
}

Recibido: 13 de julio de 2019 / Aceptado: 19 de junio de 2020

Resumen. A pesar de que la innovación social se compone de valores rupturistas, cooperativos y de superación de las soluciones preexistentes, es decir, de valores que resultan afines a las características de niños, niñas y adolescentes, estas personas no suelen ser sujetos activos de la innovación, sino solo sujetos pasivos o consumidores. Este artículo analiza los conceptos de infancia y de innovación hegemónicos que están detrás de esta situación. Con el objetivo de reforzar la efectividad del derecho de participación de niños, niñas y adolescentes en este ámbito, el artículo también propone algunos cambios en las políticas públicas para el contexto español y algunos espacios de innovación que pueden liderar este cambio. Palabras clave: Innovación social; infancia; adolescencia; políticas públicas; derechos de la infancia, políticas sociales.

\section{[pt] Inovação social e participação efetiva de crianças e adolescentes}

Resumo. Apesar de que a inovação social é composta por valores rupturistas, cooperativos e por a superação das soluções pré-existentes, a saber, por valores que estão relacionados com as características das crianças e adolescentes, estas pessoas não são geralmente sujeitos ativos de inovação, mas apenas sujeitos passivos ou consumidores. Este artigo analisa os conceitos de infância e inovação hegemônica que estão por trás dessa situação. Com o objetivo de reforçar a efetividade do direito à participação de crianças e adolescentes nesta área, o artigo também propõe algumas mudanças nas políticas públicas para o contexto espanhol e alguns espaços de inovação que podem levar a esta mudança.

Palavras chave: Inovação social; infância; adolescência; políticas públicas; direitos das crianças; políticas sociais.

\section{[en] Social Innovation and effective Participation of Children and Teenagers}

\begin{abstract}
In spite of the fact that social innovation is made up of ruptured, cooperative values and the overcoming of pre-existing solutions, that is, values that are related to the characteristics of children and adolescents, these people are not usually active subjects of innovation, but only passive subjects or consumers. This article analyzes the concepts of childhood and hegemonic innovation that are behind this situation. With the aim of reinforcing the effectiveness of the right to participation of children and adolescents in this area, the article also proposes some changes in public policies for the Spanish context and some innovation spaces that can lead to this shift.

Keywords: Social innovation; children; teenagers; public policy; children rights; social policies.

Sumario. 1. Introducción. 2. El lastre de una concepción de la infancia y la adolescencia alejada de la participación y la ciudadanía plena. 2.1. Pesimismo en el proceso de socialización. 2.2. Intensificación del modelo familiarista. 2.3. Infantilización de la esfera productiva. 3 . El lastre de una concepción estrecha de la innovación social para la infancia y la adolescencia. 3.1. Enfoques preponderantes de innovación social. 3.2. Enfoques preponderantes sobre la infancia en este escenario de innovación. 4. Hacia una innovación con participación efectiva de niños, niñas y adolescentes. 4.1. Cambios conceptuales. 4.2. Espacios de innovación a potenciar. 5. Conclusiones. 6. Referencias bibliográficas.
\end{abstract}

Cómo citar: Vila Viñas, D. (2020): Innovación social y participación efectiva de niños, niñas y adolescentes, Sociedad e Infancias, 4, 99-110. 


\section{Introducción}

Los discursos sobre innovación, bien estructurados en el interior de los sistemas de ciencia y tecnología a partir de la interacción entre las instituciones públicas, las empresas y la sociedad (Etzkowitz y Leydesdorff, 1997), bien fuera de sus límites formales, ocupan una posición privilegiada en la discusión pública y en las actividades económicas contemporáneas. Extraña por lo tanto que estos, que son discursos sustentados sobre las aspiraciones de progreso de la humanidad y sus posibilidades para construir futuros compartidos, apenas reparen en el sujeto infantil y adolescente como un agente de innovación y lo releguen al rol de campo destacado de maniobras. A lo largo del presente artículo, se buscará contextualizar esta paradoja de la innovación mediante el análisis de los enfoques sobre innovación y su relación con las concepciones predominantes sobre los niños, niñas y adolescentes. Esto permitirá proponer algunas alternativas discursivas para reforzar la agencia de tales grupos e incluso se concretarán algunas repercusiones que todo ello podría tener para las políticas públicas relativas a innovación social, la infancia y la adolescencia.

Existen algunos motivos que hacen que este objeto de investigación sea particularmente interesante para los estudios socio-jurídicos sobre infancia. En primer lugar, conviene recordar que lo habitual, en las nada numerosas investigaciones socio-jurídicas que ponen atención en la infancia y la adolescencia en España y otros Estados del sur de Europa, es que se centren en los niños, niñas y adolescentes que se sitúan en los márgenes de los procesos de socialización. Aquellos que, en el contexto de la criminología funcionalista, se denominaron grupos "desviados", por razón de la distancia entre su conducta y la norma social sancionada por el oportuno sistema jurídico (Bergalli y Bustos, 1983: 20 y ss.), por los riesgos y las necesidades de protección que surgían del funcionamiento anómalo de sus familias, o por la necesidad de reforma en la que debían implicarse instituciones educativas y correccionales. Por el contrario, los niños, niñas y adolescentes que captan la atención de los enfoques sobre innovación social no se encuentran escindidos del conjunto del cuerpo social. Ello no obsta, como se verá, para que tales discursos impongan nuevos cortes, sobre todo al hacer abstracción de desigualdades de partida relevantes. Sin embargo, en principio, los discursos y las actividades de innovación social se dirigen a la generalidad de los niños, niñas y adolescentes, en un contexto marcado por la ubicuidad de las tecnologías de la información y la comunicación (en adelante, TIC).

En segundo lugar, la propia naturaleza de la innovación social cuestiona las concepciones más habituales sobre la participación de la infancia y la adolescencia. Se trata de un concepto, el de la innovación social, que ha adquirido un vuelo creciente en el marco de las políticas europeas, de acuerdo a un imaginario que quiere destacar las oportunidad en materia de educación, culturas compartidas y convivencia democrática del continente, en contraste con las ventajas competitivas de otros bloques en la economía del conocimiento contemporánea ${ }^{2}$ y que ha ido ampliando sus componentes desde un apéndice de las políticas de investigación y desarrollo y las relativas a las TIC, hacia actividades en las que son relevantes la cooperación social y las relaciones comunitarias con saberes arraigados en los entornos en que deben tener impacto ${ }^{3}$. Si tomamos la definición de innovación social que ofrece la Oficina de Asesores y Asesoras Política Europea, como

(...) nuevas ideas (productos, servicios y modelos) que satisfacen al mismo tiempo necesidades sociales (de manera más efectiva que las alternativas) y crean nuevas relaciones sociales o colaboraciones. Dicho de otro modo, no solo son innovaciones buenas para la sociedad sino que refuerzan su capacidad para actuar (2010: 24),

se observa que la participación en estos procesos es compleja y social, basada en la agencia colectiva y no en la contribución aislada de tal niño, niña o adolescente, aunque el ejercicio efectivo de la participación en este ámbito debe garantizar las posibilidades de contribuir de cada uno de ellos y de ellas.

En tercer lugar, parecería que en este ámbito existe la oportunidad de que niños, niñas y adolescentes ejerzan una participación efectiva. En el imaginario neoliberal, el sujeto innovador se suele presentar como un agente creativo capaz de abrir ciclos económicos o disolver problemas sociales enquistados gracias a una vocación y a una audacia que contrasta con la pasividad y el comportamiento inercial de las instituciones públicas (Osborne y Gaebler, 1993), las viejas empresas (Vesper, 1990) y el cúmulo de rentistas que impiden a una sociedad desplegar sus verdaderas capacidades. Cabría pensar que el sujeto infantil pudiera emparentarse con aquel, si no fuera por la persistencia de una consideración moderna de la infancia como sujeto pasivo de intervención, protección, tutela o reforma, que entrará en tensión con este ideal contemporáneo.

Debido a las limitaciones de este trabajo, los datos y ejemplos que sustentan los análisis se referirán principalmente al contexto europeo y, dentro de este, al español, con la esperanza de que los argumentos vertidos sean razonables también para interpretar la participación efectiva de la infancia y la adolescencia en el ámbito de la innovación social más allá de estas fronteras.

El Consejo Europeo Extraordinario de Lisboa, en el año 2000, ya se subtituló con referencias a la "Europa de la innovación y el conocimiento". Ver: ttps://eur-lex.europa.eu/legal-content/ES/TXT/HTML/?uri=LEGISSUM:c10241\&from=EN.

Puede verse un resumen de la evolución reciente del concepto en los discursos de la Unión Europea, la OCDE y entornos estatales, como el británico y el canadiense, en Martínez Moreno et al. (2019: 2 y ss). En síntesis, desde una noción inicial muy ligada a la simple producción de nuevos conocimientos y tecnología en un ámbito principalmente industrial y mercantil, como la que se consagraba en el Manual de Oslo de la OCDE de 1997, se han ido incorporando nuevos agentes, espacios, procesos y resultados. 


\section{El lastre de una concepción de la infancia y la adolescencia alejada de la participación y la ciudadanía plena}

Una de las premisas de este trabajo es que las percepciones preponderantes al pensar en las relaciones entre innovación social e infancia y adolescencia se han definido socialmente y, del mismo modo, pueden redefinirse. De hecho, la delimitación de ciertas categorías sociales y sujetos es una parte constitutiva de la actividad de gobierno (Vila-Viñas, 2014a), entendida en el sentido amplio en que lo hace Foucault (2008: 93-94), como los saberes y actividades dirigidos a configurar las posibilidades de acción de los otros. En particular, la infancia ha constituido un sujeto destacado de la expansión biopolítica (Foucault, 2003: 205-207), sobre todo en la especificación de su propio ámbito (Cheal, 1991; Bell, 1993: 392) pero también, en la otra dirección, como palanca de gobierno de la familia.

Desde este punto de partida, se pueden señalar tres rasgos de la configuración contemporánea de niños, niñas y adolescentes como categoría política que resultan relevantes para comprender su escoramiento en los enfoques de participación efectiva y ciudadanía plena, así como y de manera especial para su concepción en los discursos y actividades de innovación social: la consideración pesimista del proceso de socialización, la reciente intensificación del modelo familiarista y la infantilización progresiva de la esfera productiva.

\subsection{Pesimismo en el proceso de socialización}

La tradición occidental ha sostenido el proceso de socialización de la infancia sobre una premisa pesimista. Se trata de una consideración de origen socrático, por la cual la libertad solo puede alcanzarse a través de un dominio de los propios instintos, de las pasiones naturales. De este modo, el sujeto libre sería aquel capaz de auto-dominio, de negar las pasiones que lo esclavizan y de observar en cambio las normas de la razón, de ganar esta batalla, antes exterior, en su interior (Adorno, 1992: 238, 290). Por supuesto la participación en los procesos de innovación social, como una dimensión avanzada de la inteligencia humana contemporánea, requeriría estas capacidades. Dentro de este escenario ético, niños, niñas y adolescentes aparecen como los escalones más bajos del proceso de civilización (Elias, 1987: 179 y ss.), próximos al estado de naturaleza, lejos del individuo completo, capaz de gobernarse y actuar.

De este modo, la transición hacia la plena autonomía adulta no será otra cosa que un proceso de demolición controlada de las características de lo infantil. A pesar de la democratización vivida en las últimas décadas dentro de las familias y de las instituciones escolares, este aplanamiento del campo infantil se ejecutará desde la verticalidad, la desigualdad en el acceso a los recursos y en general un enfoque disciplinario de la evolución del niño para alcanzar la edad adulta (Vila-Viñas, 2012: 547 y ss.). Todo ello es coherente con la configuración logocéntrica e individualista del acceso a la participación y a la ciudadanía, que limita los derechos políticos de grandes colectivos sociales en tanto entiende que no se ha formado en ellos un juicio suficiente para formar parte de la vida colectiva de manera plena (Gaitán, 2018: 20).

\subsection{Intensificación del modelo familiarista}

Conviene recordar que el modelo preponderante de tratamiento gubernativo de la infancia y la adolescencia en el contexto de los Estados del sur de Europa ha pivotado sobre su cuidado y socialización por parte de la familia y bajo su autoridad. A esto se ha añadido, al ritmo de los vaivenes de las políticas de inversión social más expansivas o más austeras, un conjunto de políticas en esta dirección. Por una parte, las políticas de educación universal, cuyo objetivo ha sido menos borrar la desigualdad de partida que ofrecer un común denominador civilizatorio ajustado a las especificidades de cada grupo de niños y niñas (Walkerdine, 1995: 90-91; Masschelein y Simons, 2005: 120-121). Por otra, un cuerpo de políticas familiares y de infancia de último recurso, con el objetivo de establecer ciertos mecanismos de previsión social sobre los grupos especialmente vulnerables, sobre todo cuando el cuidado en el interior de la familia se hacía inviable. Esta distribución de los esfuerzos de crianza, que Esping-Andersen (2007) denominó "modelo de políticas familiares sureuropeo", se ha visto estresada desde el momento, entre 2008 y 2010, en que se entendió que la estabilidad europea dependía del aseguramiento del pago de las deudas a los acreedores de los Estados del sur y que esto se haría principalmente mediante una reducción de la inversión social. Ello se ha traducido en un recorte del gasto de las Administraciones Públicas españolas destinado a educación no universitaria que ha tardado casi una década en aproximarse a recuperar los niveles de inversión de 2009, según la estadística de gasto público en educación. ${ }^{4}$ Todo ello sucedía al mismo tiempo que, en 2018, la proporción de personas menores de 16 que se encontraban en riesgo de pobreza en España recuperaba los niveles de 2008, aunque permanecía en un elevado 26,2\%, según la encuesta de condiciones de vida. ${ }^{5}$

En este escenario, una política pública que pretendiera dar protagonismo a la infancia en los procesos de innovación se encontraría, en primer lugar, con el obstáculo de que buena parte de las habilidades relevantes en estos procesos se desarrollan en el contexto familiar y comunitario, marcado por las tasas de desigualdad y pobreza que se han señalado y por una cierta restauración de la preponderancia familiar, al ritmo del debilitamiento de los itinerarios de des-familiarización trazados por las políticas sociales en este ámbito (Vila-Viñas, 2014b). En segundo lugar, si se quisiera vencer la transmisión intergeneracional de la desigualdad en los ámbitos familiar y comunitario con una

Fuente: Estadística de gasto público en educación. Ministerio de Educación y Formación Profesional.

Fuente: Encuesta de Condiciones de Vida 2018. Instituto Nacional de Estadística. 
política de fomento de la innovación social, por ejemplo centrada en los espacios educativos y de ocio, se debería iniciar una reconstrucción presupuestaria e institucional de estos espacios, que se han concebido con un alcance muy reducido, a la escala de las políticas de innovación social y de infancia en este contexto posterior a 2008.

\subsection{Infantilización de la esfera productive}

Durante las últimas décadas se ha desdibujado la separación entre la esfera productiva y la reproductiva que vertebraba el modelo de gobierno fordista-welfarista. Niños, niñas y adolescentes quedaban así inscritos en el ámbito familiar, el ámbito de lo reproductivo, de la acción tutelar-social del Estado y permanecían separados del ámbito productivo. Se encontraban en preparación para el mercado laboral, genuinamente liberal y reservado para sujetos autónomos completos, esto es, adultos. Tal escisión llegó por lo tanto a convertirse en un rasgo típico de modernidad y avance civilizatorio, en contraste con los Estados del sur y las economías familiares arcaicas, que, durante buena parte del siglo XX, seguían dependiendo de los aportes laborales de unos niños y niñas que no podían disfrutar de la preparación escolar en exclusiva.

En la actualidad esta cesura ha dado paso a una cierta confusión, en un movimiento que tiene dos trayectorias. De una parte, la actividad reproductiva, de crianza o de cuidados se ha consolidado como una actividad heterogénea y multidireccional, con una pluralidad de sujetos y generaciones (Finch, 1989: 30-32, 40-43; Ungerson, 1998: 172), así como de direcciones (Millar, 2009: 114, 117 y ss). Entre otras investigaciones, la literatura citada muestra que la producción intrafamiliar no fluye solo de madres y padres a niños y niñas y que no se trata de una actividad simple. Al tiempo que se constata que estas relaciones interiores en torno a la crianza son complejas, también se advierte un aumento de las exigencias de esta actividad y de la participación de agentes privados en régimen de mercado con el objeto de prestar servicios de atención personal. Al mismo tiempo que se producía este movimiento, las cualidades propias del ámbito de los cuidados y la crianza han cobrado valor en el ámbito productivo, no solo como la citada actividad específica de servicios personales provistos por el mercado, sino en el contexto de una posfordización de las relaciones laborales y las políticas sociales y del devenir antropogenético de la producción, esto es, de la producción de humanidad por parte del ser humano a través de sus competencias emocionales, lingüísticas, culturales y relacionales (Marazzi, 2003), que es particularmente visible en el ámbito de la innovación social. Es decir, el estándar infantil y adolescente pasa a estar cada vez más inscrito en la racionalidad productiva en distintos órdenes sociales, lo que contrasta con el escoramiento de su participación efectiva en el ámbito de la innovación social.

\section{El lastre de una concepción estrecha de la innovación social para la infancia y la adolescencia}

A pesar de que la innovación exige un abordaje rupturista de los problemas sociales, sus enfoques predominantes no están revirtiendo la subordinación de niños, niñas y adolescentes en este ámbito. En esta sección se buscará explicar las dificultades para este avance desde la particular configuración del ámbito de la innovación y desde la posición que ocupa ahí la infancia y la adolescencia.

\subsection{Enfoques preponderantes de innovación social}

Si se examina con detenimiento cuáles son los enfoques de innovación preponderantes, resulta más sencillo identificar las dificultades para asegurar que la infancia y la adolescencia tengan una agencia plena en este ámbito. En esta dirección se considerará la preponderancia de los regímenes de mercado, de un sujeto de innovación individualizado y autónomo y una jerarquización de los saberes contraria a los más frecuentes en niños, niñas y adolescentes.

Para ello es importante, en primer lugar, hacer descender el análisis desde las definiciones generales, que tienen un elevado componente normativo, hacia los regímenes de prácticas en que el conocimiento se usa para resolver o abordar de manera rupturista y aportando valor social determinados problemas sociales. La definición de innovación social de la que se ha partido en la introducción de este artículo hacía hincapié en el valor social de la creación y en las relaciones de colaboración que generaba o fortalecía. En la práctica, la imposición de una racionalidad de mercado condiciona mucho más la innovación de lo que a los organismos y think tanks internacionales les gusta reconocer. No solo porque ese es el contexto que condiciona variables tan relevantes como el tiempo disponible, la capacidad organizativa y los saberes técnicos de las comunidades, sino sobre todo porque marca los parámetros de sostenibilidad, en el sentido amplio de posibilidades de financiación o escalabilidad, de las innovaciones que pudieran emerger. En la práctica es frecuente que el criterio de validación principal no sea el valor social que pueda producir la innovación, sino la tasa de beneficio que pueda asegurar a quien está en disposición de financiarla. En contextos aún más inscritos en racionalidades de mercado como los propios de la economía del conocimiento, en los que éste se convierte en el factor principal de la producción (Moulier-Boutang, 2011), la subordinación a tales criterios será aún mayor. Por supuesto, existe una pugna entre esta concepción y otras perspectivas alternativas de la innovación (Leadbeater, 1997; Moulaert et al., 2010, entre otras referencias), en las que resulta previsible que, al basarse en el protagonismo de la sociedad civil, niños, niñas y adolescentes tuvieran un rol más relevante. Sin embargo, la cuestión sigue siendo 
la distancia entre el plano normativo y la descripción del contexto en que se inscribe este artículo ${ }^{6}$. La citada subsunción de la innovación en los términos del mercado no excluye de forma necesaria la participación de niños, niñas y adolescentes, ya que, como se verá, son sujetos relevantes en estas economías contemporáneas, pero sí hará difícil que puedan ser agentes, sujetos capaces de dirigir con autonomía su participación y la orientación de esos procesos, en tanto su poder específico en el mercado es menor.

En segundo lugar, el rol que los enfoques emergentes sobre innovación conceden al conocimiento se compatibiliza mal con un incremento de la agencia de niños, niñas y adolescentes. Como se señaló, el acceso al conocimiento suele identificarse con un proceso de adquisición de gobierno de sí y autonomía respecto a las pasiones naturales y a las exigencias del entorno social (la tradición, la comunidad, la autoridad...), que se corresponden bien con el proceso de civilización-individuación, pero peor con otras formas de adquirir conocimiento y aplicarlo a la resolución de problemas sociales que son frecuentes entre niños, niñas y adolescentes. En estas etapas es más habitual el uso de saberes encarnados en la existencia de unos lazos sociales y de unas relaciones de interdependencia densas. Si solo el sujeto individual y autónomo puede ser agente de innovación, solo el adulto (y tampoco todas las personas adultas) está llamado a ocupar esa posición.

En tercer lugar, uno de los efectos de aplicar a las prácticas de innovación un régimen de veridicción de mercado, es decir, dar por verdaderas, por innovadoras, aquellas que funcionan ahí y descartar las que no, es que se introduce una jerarquización de los saberes que hace prevalecer los conocimientos técnicos, largamente formados en el interior de esos regímenes de mercado, respecto a otros que pueden ser más frecuentes en las capas infantiles y adolescentes. Como Lewis Mumford señalaba este proceso no es nada natural:

Lo cualitativo quedó circunscripto a lo subjetivo, lo subjetivo fue descartado como irreal, y lo que no podía verse o medirse, como no existente. La intuición y el sentimiento no ejercieron influencia en los procedimientos o en las explicaciones mecánicas. La nueva ciencia y la nueva técnica podían realizar una obra vasta porque mucho de lo que estaba asociado con la vida y con el trabajo en el pasado — el arte, la poesía, el ritmo orgánico y la fantasíahabía sido deliberadamente eliminado. A medida que el mundo exterior de la percepción adquiría mayor importancia, el mundo interior del sentimiento se reducía. La división del trabajo y la especialización en partes aisladas de una operación, que ya habían comenzado a caracterizar la vida económica del siglo XVII, prevalecieron en el mundo del pensamiento; fueron expresiones del mismo deseo de obtener precisión mecánica y resultados rápidos (Mumford, 2009: 85).

Una innovación más afín a las formas de funcionamiento y saberes acumulados en las comunidades infantiles y adolescentes debería apoyarse más en las llamadas tecnologías blandas ${ }^{7}$, estas son los protocolos sociales, organizativos y técnicos que permiten hacer efectivas las transformaciones sociales y las rupturas de las tecnologías que ocupan de manera tradicional el imaginario sobre la innovación. Esta inversión jerárquica abriría el espacio al protagonismo de las comunidades que manejan estos saberes desde una alta inserción en sus territorios y espacios sociales y desde los lenguajes y prácticas que les resultan propios. Ello no resuelve de manera directa la participación de niños, niñas y adolescentes, ya que las relaciones adultocráticas estructuran también esas comunidades, ni supera la necesidad de concretar más la investigación para determinar cuáles son los medios más adecuados para facilitar la participación de niños, niñas y adolescentes en la innovación pero daría paso a escenarios de mayor movilidad en las relaciones de poder que los habituales en una economía del conocimiento centrada en exclusiva en el mercado.

En cualquier caso, esta jerarquía de saberes dentro del ámbito de la innovación, así como el conjunto de los marcos discusivos que rigen en este ámbito, deben situarse en un contexto general en el que no solo éstas, sino el conjunto de las políticas de $\mathrm{I}+\mathrm{D}+\mathrm{i}$ han estado subordinadas al pago a los acreedores y la reducción de las inversiones productivas. En términos presupuestarios, mientras durante el periodo crítico 2009-2016 el aumento de la inversión en I+D en Reino Unido o Alemania estuvo cerca del 40\% (39,3\% y 37,9\% respectivamente) e incluso aumentó un $12,5 \%$ en Italia, se redujo en España en un 9,1\%, según datos del INE y de Eurostat. Considerando el conjunto de las políticas de $\mathrm{I}+\mathrm{D}+\mathrm{i}$, se observa cómo no han llegado a movilizar recursos para alcanzar ni siquiera el umbral del 1,2\% del PIB, lo que las sitúa lejos del objetivo del 2\%, al que sí ha llegado la media de la Unión Europea, siendo el que vertebraba los objetivos de su Horizonte 2020. A esta baja previsión debe añadirse el mantenimiento de tasas de ejecución de lo previsto en los presupuestos generales del Estado extraordinariamente bajas, que, por ejemplo, no llegaron al 30\% en 2017 (29,7\%) ni a la mitad de lo presupuestado en 2018 (46,8\%), según datos de la Intervención General del Estado.

\footnotetext{
Cuando Martínez Moreno et al. (2019) han analizado la realidad de la innovación social en Cataluña identifican esta distancia entre ambos planos: "A partir de estas ideas y del trabajo empírico realizado, se hace necesario pensar en la composición social de estas prácticas. La metodología cartográfica empleada, basada en el contraste entre las pautas de distribución territorial de la innovación social y las dinámicas de segregación socio-espacial, nos permite enfatizar en una idea habitualmente desconsiderada en otras investigaciones basadas en estudios de caso, a saber: que no innova quien más padece la crisis o quien más lo necesita, sino quienes, sobre todo, tienen más recursos para poder organizarse. Las iniciativas se concentran en los territorios donde viven personas con perfiles que, si bien han padecido la crisis, también tienen recursos cognitivos, culturales y organizativos para construir respuestas. La capacidad de acción colectiva, significativamente condicionada por los recursos socioeconómicos y sobre todo socioeducativos, parece ser un factor de gran importancia para la innovación social" (pp. 14-15).

Puede ampliarse este concepto, en su contraposición con la tradicional hegemonía de las tecnologías duras en el ámbito global de la economía basada en conocimiento en Jin (2011) y en el de la innovación social más centrada en comunidades en el Hirikilab (2015).
} 


\subsection{Enfoques preponderantes sobre la infancia en este escenario de innovación}

A este escenario, niños, niñas y adolescentes arrastran algunas de las características que definen su posición social de una manera general. En primer lugar, su posición se considera exterior a los procesos productivos (Wintersberger, 1994: 74-75). Se suele admitir que esta posición es relevante en las decisiones de consumo e incluso en el diseño de los productos, como Juliet Schor (2006: 20-29) demostró con respecto al estrato de niños y niñas entre los 6 y los 12 años de edad, pero no que ellos sean sujetos activos de los procesos productivos. Además, su rol en los procesos de consumo de las TIC se encuentra reforzado, toda vez que actúan como la puerta de entrada de muchos productos y servicios al interior de las instituciones y las familias. Literatura reciente sobre investigaciones sobre niños y niñas con destino al marketing o a la prestación de servicios educativos y de atención constata esta intensificación. Por ejemplo, Davidson et al. (2018: 95-96) señalan distintos estudios realizados a través de la grabación con cámaras insertas en ropa y utensilios (wereables) que las criaturas utilizan de manera cotidiana para que quienes investigan puedan adoptar su punto de vista influyendo menos en su comportamiento. A pesar del uso generalizado de las TIC por parte de niños, niñas y adolescentes, en estos enfoques resulta evidente que su posición no es autónoma, sino pasiva, sobre todo como fuente de información y de datos para las investigaciones (Deli-Gray et al., 2016) o a la manera clásica de quienes influyen en las decisiones de consumo de las personas adultas. Eventualmente el uso que hacen de las TIC puede resultar muy conveniente para su bienestar o el interés general, pero en todo caso las innovaciones que puedan producirse lo harán lejos de su participación y dirección.

Como se indicó, parte de la paradoja de la innovación social es que niños, niñas y adolescentes cada vez se consideran más relevantes en los procesos de diseño y consumo y en el ciclo de la innovación, dentro y fuera del mercado, pero no acceden a ser protagonistas de estos procesos, a tener una participación efectiva en los mismos. Este desequilibrio ya formaba parte de los discursos sobre la contribución de la infancia y la adolescencia a las actividades productivas que se estructuraron en torno a los enfoques sobre el capital humano. La citada consideración de la infancia como un futurible, un estrato al que gobernar a la luz de su proceso de constitución en un sujeto completo a la edad adulta encontró un punto de confirmación al hibridarse con los enfoques sobre el capital humano (Jenson, 2006: 33; Palley y Shdaimah, 2011: 1159). La posibilidad de hacer de las personas un objeto de inversión puesto al servicio de la expansión de los procesos productivos, esto es un capital, cobraba especial sentido cuando se refería a los niños y niñas, y a la rentabilidad de las políticas educativas y de atención a la primera infancia. El consenso sobre las oportunidades de una economía basada en el conocimiento y la innovación para convertirse en uno de los pocos ámbitos rentables en los entornos postindustriales ha reforzado este enfoque. Huelga decir que, aunque estas perspectivas de capital humano puedan establecer una prioridad estratégica en las inversiones sobre la infancia, la posición de niños y niñas es la de objeto de tales inversiones y activo de futuro en un cálculo de las fuerzas del Estado mucho más próximo a enfoques de policía, en sentido clásico (Foucault, 2008: 297-298), que del acceso de niños, niñas y adolescentes a la ciudadanía plena.

Vista la preeminencia de estos enfoques adultocráticos, tampoco extraña que la forma de invertir en la infancia para potenciar el capital humano sea poblar los procesos educativos de gadgets tecnológicos, anticipar el acceso de niños y niñas a internet y a smartphones sin un programa educativo eficaz para que las nuevas generaciones se muevan en los reinos de la hiperconectividad, la memoria digital de sus acciones y la extracción de datos. Esto es coherente con la jerarquización de saberes y el reduccionismo de los procesos de innovación que se indicaron y, como ha ocurrido en otras ocasiones en las que el foco se ha puesto en los niños, niñas y adolescentes, viene acompañado de una mayor responsabilización de estas personas por su destino en el nuevo escenario.

\section{Hacia una innovación con participación efectiva de niños, niñas y adolescents}

Hasta aquí se han expuesto los motivos, algunos de profundo arraigo en la configuración de nuestras sociedades, otros más coyunturales, que explican lo que se denominó la paradoja de la innovación respecto a la participación de niños, niñas y adolescentes. Es decir, su extraordinaria relevancia como sujetos que contienen los valores disruptivos y de futuro, anidados a la concepción de las actividades basadas en la innovación, al mismo tiempo que su orillamiento como agentes protagonistas de tales procesos. Los argumentos anteriores deberían haber servido para desnaturalizar estas concepciones, al tiempo que ahora conviene realizar algunas propuestas para revertir esta situación. Para ello se apuntarán primero algunos presupuestos conceptuales que deberían cambiar para poder pensar y gobernar la innovación de otro modo y, a continuación, se señalarán ciertos espacios en los que es previsible que esto resulte más viable, de modo que se designen como espacios prioritarios de innovación social con protagonismo de niños, niñas $\mathrm{y}$ adolescentes.

\subsection{Cambios conceptuales}

La primera recomendación es considerar la participación de la infancia en las actividades de innovación social como una expresión de su ciudadanía plena, en la propuesta de ciudadanía que estructura Gaitán (2018: 24 y ss.) como práctica, no vinculada a estatutos normativos ni a situaciones abstractas a las que se accede en determinado punto 
del "desarrollo" vital, sino como un conjunto de prácticas cotidianas basadas en un haz de relaciones entre personas diferentes que tienen sin embargo la misma posibilidad de ser parte de esas actividades o de esa búsqueda de soluciones desde su singularidad.

Por supuesto, el ejercicio efectivo de cualquier derecho requiere de intervenciones públicas y sociales destinadas a hacerlo posible, a configurar su marco de posibilidad, a veces mediante normas jurídicas, a veces mediante el levantamiento de dispositivos completos, como el sistema educativo y otras manifestaciones de los servicios públicos. Aunque el diseño de intervenciones concretas escapa de la capacidad de este artículo, sí resulta claro que deben existir intervenciones públicas positivas orientadas a mejorar la igualdad de oportunidades y que este enfoque se encuentra alejado de aquellos discursos y políticas de innovación que preparan a niños, niñas y adolescentes para innovar mañana, cuando sean personas adultas, desde la premisa de que solo entonces podrán tener una participación digna de tal nombre en esos asuntos.

La segunda recomendación es modificar el sujeto arquetípico de la innovación para hacerlo permeable a características propias de la manera de estar en el mundo de niños, niñas y adolescentes, que son al tiempo rasgos afines a la ética de la innovación. Si ésta se piensa menos como avances en saberes $\mathrm{STEM}^{8}$, ceñidos a una actividad individual u organizada de manera jerárquica y que deben encontrar además validación en el mercado, para pensarla en cambio como una solución colectiva y rupturista, que aporta nuevo valor social al abordaje de problemas sociales o situaciones cotidianas, será más sencillo integrar las características de maleabilidad, colaboración, sentido lúdico y enfoque disruptivo que son frecuentes en niños, niñas y adolescentes.

La tercera recomendación es democratizar el régimen de funcionamiento de las instituciones sociales en las que se desarrolla la mayor parte de la vida de niños, niñas y adolescentes, tales como la familia o la escuela. A lo largo de las últimas décadas, los llamados Childhood Studies han mostrado que niños y niñas son sujetos sociales, en tanto resultado de su inscripción en instituciones, estructuras sociales y relaciones con sujetos y grupos de distintas generaciones, géneros, estratos sociales y etnias, dentro de un marco adultocrático (Qvortrup, 2007; Wintersberger y Olk, 2007), al tiempo que también son sujetos dotados de agencia, con capacidad de intervenir en esas relaciones y estructuras (James, 2009: 40-43; Mayall, 2009). En este escenario, por ejemplo, se observan evidencias de su protagonismo en la transformación de las propias comunidades (Burch, 2014) y en la transmisión de saberes tecnológicos dentro de la familia (Correa, 2014).

A pesar de esta evolución, las principales instituciones de socialización de la infancia persisten en el esquema socrático de socialización: control de las pasiones por la razón. Un camino de auto-gobierno a través de la borradura de los rasgos infantiles que será condición imprescindible para constituirse en sujeto libre y por lo tanto poder participar de la ciudadanía plena y sus actividades asociadas. Civilizar será sacar de un estado de "minoría de edad", como "cierto estado de nuestra autonomía de la voluntad que nos hace aceptar la autoridad de algún otro para conducirnos en los dominios en los que es conveniente hacer uso de la razón” (Foucault, 2002b: 85). Como ocurre en el arte, solo tras el dominio del canon se puede emprender el viaje de la trasgresión, en lo que es el núcleo de la paradoja de la participación de la infancia en la innovación: innovar exigirá utilizar la mayor cantidad de rasgos "infantiles” o "salvajes", que deberán en cualquier caso sobrevivir a un proceso de socialización orientado a borrarlos. Por lo demás, no cabe duda de que la democratización de estas instituciones favorecería un entorno de producción horizontal basada en comunes, que correría en paralelo a los procesos de democratización de la innovación y la producción contemporánea que ha descrito, entre otros muchos, Yonkai Benkler (2006).

La cuarta recomendación es superar la racionalidad neoliberal que predomina en el régimen actual tanto de la innovación como de la participación de niños, niñas y adolescentes. Si bien se ha mostrado el peso de la infancia en los enfoques centrados en el capital humano, en la medida en que su surgimiento se ha correspondido con el ataque a los dispositivos abstractos de solidaridad e igualación social propios del welfare durante las últimas décadas, esto ha venido acompañado de un aumento en la desigualdad en la infancia ${ }^{9}$ y un deterioro del rol de los sistemas educativo y de tiempo libre en la reducción de esas brechas sociales (Asiego y Ubrich, 2015). Sin revertir esa tendencia es imposible imaginar que se abra de manera efectiva el acceso a las actividades de innovación social.

Por situar esta cuestión en nuestro ámbito, conviene señalar que recientemente Xie et al. (2019) publicaban su investigación sobre cómo los sistemas de inteligencia artificial alojados en robots podían ayudar al desarrollo de los niños y niñas chinos que habían quedado en el medio rural mientras sus padres emigraban a otras zonas del país a trabajar. Tanto los resultados obtenidos, como la cantidad de aplicaciones análogas que citan, muestran el punto crítico en que se encuentran las políticas de infancia en este contexto: en la bifurcación entre la delegación de las actividades de crianza más básicas (como aprender a hablar o escuchar cuentos y canciones) a empresas tecnológicas y la incorporación de la tecnología al servicio de los dispositivos de igualación social. Es decir, si niños y niñas van a ser clientes y proveedores de datos para perfeccionamiento de sistemas privados de inteligencia artificial, en el contexto

\footnotetext{
Acrónimo formado por la traducción inglesa de las palabras "ciencias, tecnología, ingeniería y matemáticas".

En 2018 la tasa de personas menores de 16 años en riesgo de pobreza (renta inferior al 60\% de la mediana) en España se sitúo casi 5 puntos por encima $(26,2)$ de la correspondiente al total de la población $(21,5)$, según la Encuesta de Condiciones de Vida del INE de 2019. Si, en el mismo país, se compara la tasa de pobreza entre menores de 16 años y mayores de 64 en 2016, estableciendo una ratio entre ellas, se obtiene un valor de 2,44 lo que significa que, en proporción, existen casi dos veces y media más personas en riesgo de pobreza entre las menores de 16 años que entre las mayores de 64 y esta proporción ha subido casi se ha doblado en el conjunto de la UE-27 y se ha multiplicado por más de tres en países como España y Grecia o casi por tres en Francia entre 2005 y 2016 (Fernández, 2017).
} 
de descomposición de las instituciones sociales más básicas, o van a ser protagonistas, crecientemente empoderados, en estos procesos, al margen de su situación de partida por la familia en que hayan nacido.

En este sentido, la manera en que la desigualdad que afecta a la infancia se replica en los usos que pueden hacer de las TIC no es esperanzadora. En 2017 Unicef alertaba de que el desigual acceso a las TIC estaba abriendo nuevas brechas entre niños, niñas y adolescentes. Las políticas sociales y educativas tienen la llave. Desde luego, las que no están orientadas hacia el aumento de la inclusión social no pueden aspirar a ampliar la participación de la infancia en la innovación. El modelo de Parziale y Scotti (2016) muestra cómo un aumento de la inversión social en educación dirigida a aumentar sus efectos de inclusión social puede reducir la desigualdad e impactar positivamente sobre el crecimiento. Junto a otras políticas sociales, esto puede ampliar los recursos cognitivos, a escala individual y colectiva, y mejorar el ambiente cultural de los entornos educativos y familiares; elementos asimismo básicos en los procesos de innovación social.

\subsection{Espacios de innovación a potenciar}

Repasados los factores determinantes de la citada paradoja de la innovación e identificados algunos cambios que sería imprescindible operar en las concepciones de partida y en las políticas públicas, resulta oportuno señalar ámbitos que pueden liderar su disolución y hacer efectivo el derecho a la participación en este campo.

En 2017, como parte del lanzamiento del proyecto $\mathrm{PEEL}^{10}$, cuyo objetivo es favorecer que niños, niñas y jóvenes tengan voz en la configuración de su identidad digital, se celebró una mesa redonda para discutir las políticas que harían posible la efectividad de sus derechos en la era digital, a partir de la premisa de que niños, niñas y adolescentes no pueden ser simples objetos o receptores de las políticas educativas y digitales, sino que deben conformarlas. Uno de los ámbitos en que se manifiesta con más claridad esta tensión es en el de producción y uso de datos. Por una parte, es imprescindible que los niños, niñas y adolescentes participen activamente en el diseño de nuevas instituciones sociales o dispositivos (Runciman, 2017: 2). Sin embargo, aquéllos deben poder comprender cuál es la naturaleza de su participación, cómo se están usando sus datos, qué rol van a desempeñar en estas nuevas instituciones o dispositivos, hacerlo en un entorno seguro en el que poder expresarse con libertad y conservar siempre el poder de consentir o no con estas prácticas. Si esto es así, se abren un sinfín de oportunidades para desarrollar herramientas educativas que fomenten la cooperación y el empoderamiento en el campo de la innovación social desde los primeros años, que permitan articular estos aprendizajes y prácticas con el conjunto de la comunidad educativa, incluidas las familias e instituciones comunitarias, y conquistar autonomía respecto a la dirección exclusiva de los gigantes tecnológicos.

Otro ámbito donde es más viable que prolifere la participación de niños, niñas y adolescentes es en el del conocimiento abierto, esto es, el de los espacios de producción cognitiva, muchas veces próximos a la innovación, en los que priman las libertades de acceso, uso, réplica y modificación de los saberes y sus manifestaciones concretas (Stallman, 2004; Lessig, 2006; Benkler, 2006). Estos proyectos se caracterizan por eliminar las restricciones de acceso al uso del conocimiento. Por ejemplo, son experiencias trasladables a la preocupación de este artículo las de proyectos como Wikipedia, una enciclopedia de producción y consultas abiertas y de edición-curación horizontal, que permitiría a niños, niñas y adolescentes pasar de ser siempre receptores de contenidos creados por otras personas adultas a, creadores (Bissell y Boyle, 2007), algo que es común a muchos recursos educativos en abierto (Vila-Viñas et al., 2015).

Desde saberes próximos a la ingeniería y la programación, el proyecto Arduino es un ejemplo constante de innovación abierta a la participación. Arduino es hardware libre, esto es, un tablero microcontrolador simple y un ambiente de desarrollo para escribir software en él (Pearce, 2014; Vila-Viñas et al., 2015). Esto permite crear con facilidad dispositivos y ambientes interactivos sin las restricciones habituales de la propiedad industrial, de modo que, dentro de la variedad de licencias libres existentes, este material puede modificarse y, a partir del mismo, crearse nuevos diseños, productos y documentación sin restricciones. La modularidad del hardware libre ha permitido que este proyecto y otros análogos, como Rasperri $\mathrm{Pi}$, se orienten a niños, niñas y adolescentes, configurando entornos adecuados para su participación en la innovación, no solo cuando estas herramientas se utilizan para crear dispositivos útiles en los procesos educativos (Lupetti, 2017), sino también cuando se implica a niños, niñas y adolescentes en su diseño ${ }^{11}$. Es habitual que el uso colaborativo de hardware libre se combine con la creación o modificación de software específico para esos dispositivos. El ámbito del software libre es señalado con frecuencia como uno de los campos más eficaces de las formas de producción horizontal basadas en el conocimiento libre (Stallman, 2002). Dadas las dinámicas de trabajo comunitario y la ausencia de restricciones de acceso propias de este ámbito, existe la oportunidad de incorporar a su desarrollo a niños, niñas y adolescentes.

Sin embargo, los espacios más propicios para la innovación no son solo tecnológicos. También aparecen cuando se consigue vadear algunas de las restricciones predominantes en determinadas prácticas sociales. Por ejemplo, el camino hacia la escuela puede ser un ambiente propicio si se organizan rutas escolares a pie supervisadas por la

\footnotetext{
Ver https://projectpeel.org/ para más información.

1 En la web del proyecto Arduino (https://create.arduino.cc/projecthub/projects/tags/kids) se pueden ver iniciativas, de distinta complejidad, para la realización con niños, niñas y adolescentes. Desde procesos sencillos de encendido sincronizado de semáforos en una maqueta a otros de construcción de robotos o impresión en 3D más complejos. Es habitual que estos proyectos se desarrollen en espacios de ocio, como campamentos durante las vacaciones, a veces incluso con una orientación inter-generacional, buscando incorporar ese diálogo de saberes, como el proyecto "Yes, I Camp. Campamento Intergeneracional" (https://www.lamolinera.net/).
} 
comunidad $^{12}$, en lugar de trayectos atomizados en automóvil. Esto modifica las relaciones habituales de cuidado, descentra la posición de la familia y permite que niños y niñas fortalezcan las relaciones entre iguales y establezcan nuevas relaciones con los adultos que forman parte del tejido social y comercial de los barrios. Como es habitual, niños y niñas son cuidados, pero también imaginarán soluciones para el cuidado de su entorno, al dotarlo de una vida y de unas conexiones distintas a las de los trayectos en coche.

Por otra parte, las actividades agrarias y otras actividades de alto componente tradicional pueden ayudar a establecer relaciones de cooperación intergeneracionales que cortocircuiten la segmentación etaria y con ello el funcionamiento adultocrático de nuestra sociedad. En los últimos años, muchas ciudades han incorporado o regenerado espacios agrícolas en su interior o en su entorno para potenciar usos de lo que generalmente se conoce como agricultura tradicional, familiar o campesina (Houtart, 2015), según el contexto. Aunque buena parte de estas explotaciones están destinadas a la producción de alimentos dentro del mercado, de manera que se haga sostenible un modo de producción que aporta un gran valor de uso, hay una proporción que permite emprender proyectos en los que niños, niñas y adolescentes (Richter y Cuenca, 2018; Quesada y Matas, 2018) pueden compartir conocimientos tradicionales y participar en la producción y gestión de estas huertas, en el contexto de una relación distinta a la habitual verticalidad del sistema educativo y, en definitiva, propicio para la innovación social (Moreno Valle, 2018; Ginés, 2019).

En la misma clave, modificar dinámicas seculares en las formas de socialización de la infancia también puede abrir espacios para la innovación protagonizada por niños, niñas y adolescentes. Es habitual que la innovación en el terreno educativo se centre en la actividad dentro del aula, los materiales y las metodologías docentes, o en las comunidades pedagógicas que pueden articularse en la periferia de la educación reglada (Vila-Viñas et al., 2015) pero, en los últimos años, se ha problematizado la disposición de los patios de recreo de las escuelas y la influencia que esto tenía sobre un ocio dominado por el fútbol, practicado sobre todo por los niños más mayores, en detrimento de otros usos. ${ }^{13} \mathrm{Al}$ modificar el espacio y regular el juego de manera más inclusiva, han podido emerger, no sin conflictos ni componentes paternalistas, otras formas de juego e interacciones, lo que dispone el terreno para que puedan germinar las innovaciones. De hecho, el juego se perfila como un espacio adecuado para la innovación protagonizada por niños, niñas y adolescentes. Puede parecer un caso excepcional, pero Covington (2018) refiere la historia de un niño de Minneapolis, de 10 años, que desarrolló un juego inspirado en el ping-pong a partir de la experiencia de su hermana mayor, que debido a su autismo no podía jugar a la versión original.

Como última fuente de ejemplos, las experiencias de divulgación científica y de incorporación de una ciudadanía sin especial preparación técnica a la actividad científica (ciencia ciudadana), entre la que se incluyen niños, niñas y adolescentes, también ofrecen buenos ejemplos de procesos de innovación social en los que éstos pueden ser protagonistas. Estos proyectos pueden crear, por ejemplo, a partir de los aportes de sus habitantes, mapas con los puntos de mayor contaminación acústica, del aire o de las calles (Fab Lab Barcelona, 2018), de manera que sirvan para mejorar los servicios de limpieza o las decisiones urbanísticas.

\section{Conclusión}

Al considerar la efectividad del derecho de participación de niños, niñas y adolescentes en el ámbito de la innovación social, este artículo partía de la constatación de la que ha denominado paradoja de la innovación, en virtud de la cual un campo dominado por valores anidados en los enfoques rupturistas de las poblaciones más jóvenes apenas ofrecía espacio para la participación de quienes es plausible que encarnen esos valores a menudo, como son los niños, las niñas y los adolescentes. Para analizar los motivos de esta exclusión se ha considerado, por una parte, los regímenes de tratamiento de la infancia y, por otra, de la innovación social. En cuanto a la infancia y adolescencia, se afirma, en primer lugar, que sigue pesando una premisa pesimista en el tratamiento de la infancia, de modo que en el presente solo puedan operar como consumidores y sujetos pasivos de la innovación, posponiendo su intervención a un futuro en que se hayan superado sus formas de estar infantiles y adolescentes. Y, en segundo lugar, que el proceso de deterioro del Estado del bienestar ha reforzado el régimen familiar en los procesos de socialización de la infancia, en detrimento de instituciones en las que la herencia familiar no se traslada de manera tan directa a las nuevas generaciones. Este es el contexto en que muchos niños, niñas y adolescentes de familias con menos capital cultural y acceso a las TIC tienen mayores dificultades para participar de manera efectiva en estos ámbitos. En cuanto a la configuración social de la innovación, para explicar esta exclusión de niños, niñas y adolescentes, se observa que su concepción hegemónica está demasiado inscrita en los regímenes de mercado, demasiado ceñida al sujeto individual como sujeto generador de conocimiento, los saberes tecnológicos vinculados a lo STEM son prevalentes en exceso y las políticas de $\mathrm{I}+\mathrm{D}+\mathrm{i}$ se encuentran en España en franca retracción.

A partir de este análisis, se proponen dos líneas de trabajo hacia la efectividad de los derechos de participación de niños, niñas y adolescentes en este ámbito. En primer lugar, se proponen algunos cambios en estas políticas públicas,

12 En los últimos años, muchos Ayuntamientos españoles han impulsado estas rutas bajo la denominación de rutas escolares seguras o caminos escolares.

13 María Zuil (2019) expone en un reportaje alguno de los casos más relevantes en el contexto español. En la documentación del proyecto de Basurama (http://basurama.org) en el CEIP Cristóbal Colón de Villaverde (Madrid) se observa un proceso de participación que cuenta con el profesorado, madres, padres, niños y niñas para la transformación de tres espacios de juego en la zona infantil. Véase asimismo el proyecto MICOS, sobre rediseño participativo de patios de colegios públicos, en Madrid (http://madridsalud.es/wp-content/uploads/2016/04/MICOS-Regeneracion-urbana.pdf). 
como considerar a las comunidades de niños, niñas y adolescentes como sujetos de innovación como tales; aproximar las concepciones de innovación a rasgos más colectivos, menos inscritos en el mercado y más cercanos a las prácticas habituales de estos sectores; $y$, por último, incidir en la democratización de las instituciones de socialización de estos grupos, particularmente en la familia y los entornos educativos, de manera que esos espacios se rijan más por los criterios de apertura, horizontalidad y prevalencia del talento que son propios, al menos desde una perspectiva normativa, de los entornos de innovación social. En segundo lugar, se indican algunos sectores concretos, tanto en ámbitos vinculados a las TIC como más allá, por ejemplo, relativos a la organización escolar, en los que estos cambios ya tienen cierta presencia y se presumen por lo tanto espacios privilegiados para reforzar la efectividad de este derecho de participación de niños, niñas y adolescentes en el ámbito de la innovación social.

\section{Referencias bibliográficas}

Adorno, T. W. (1992). Dialéctica negativa. Madrid: Taurus.

Asiego, V., Ubrich, T. (2015). Iluminando el futuro. Madrid: Save the Children (en línea). https://www.savethechildren.es/sites/ default/files/imce/docs/pobreza-equidad-educativa-espana_iluminando-el-futuro.pdf, acceso 12 de julio de 2019

Bell, V. (1993). Governing childhood: neo-liberalism and the law. Economy and society, 22(3), 390-405.

Benkler, Y. (2006). The Wealth of Networks: How Social Production Transforms Markets and Freedom. New Haven: Yale University Press.

Bergalli, R., Bustos Ramírez, J. (1983). El pensamiento criminológico. Barcelona: Península.

Bissell, S., Boyle, J. (2007). Towards a Global Learning Commons: ccLearn. Educational Technology, 47(6), 5-9.

Burch, G. W. (2014). Child Protagonism in Transformational Community Development. Transformation, 31(1), 36.

Bureau of European Policy Advisors. (2010). Empowering People, Driving Change. Social Innovation in European Union (p. 130) (en línea). https://ec.europa.eu/migrant-integration/librarydoc/empowering-people-driving-change-social-innovation-in-the-european-union, acceso 12 de julio de 2019.

Carr, K., Kendal, R. L., Flynn, E. G. (2015). Imitate or innovate? Children's innovation is influenced by the efficacy of observed behaviour. Cognition, 142, 322-332. 10.1016/j.cognition.2015.05.005

Cheal, D. (1991). Family and the State theory. New York: Havester Wheatsheaf.

Correa, T. (2014). Bottom-Up Technology Transmission Within Families: Exploring How Youths Influence Their Parents' Digital Media Use With Dyadic Data. Journal of Communication, 64(1), 103.

Covington, H. (2018). Boy Invents Game To Help Sister With Autism. Palaestra, (2), 54.

Davidson, C., MacDonald, A., Fenton, A. (2018). «These are my camera glasses»: Wearable Digital Video Glasses for Recording and Examining Young Children's Social Interactions. Asia-Pacific Journal of Research in Early Childhood Education, 12(3), 93.

Deli-Gray, Z., Pinto, M.-P., McLaughlin, C., Szilas, R. (2016). Perception of young children of the ideal shopping experience. International Journal of Retail \& Distribution Management, 44(10), 996.

Elias, N. (1987). El proceso de la civilización: investigaciones sociogenéticas y psicogenéticas. México DF: FCE.

Esping-Andersen, G. (2007). Children in the welfare state. En G. Esping-Andersen (Ed.), Family formation and family dilemmas in contemporary Europe (pp. 223-263). Bilbao: Fundación BBVA.

Etzkowitz, H., y Leydesdorff, L. (1997). Universities in the Global Knowledge Economy: A Triple Helix of University - Industry - Government Relations. London: Pinter.

Fab Lab Barcelona. (2018). Revolución ciencia ciudadana. The Making Sense EU Project [Documental] (en línea). https://www. youtube.com/watch?v=hvn5LyACUYw, acceso 12 de julio de 2019.

Fernández, J. J. (2017). Evolución comparada de la pobreza infantil, juvenil y de los mayores en Europa. Observatorio Social de la Caixa (en línea). https://observatoriosociallacaixa.org/es/-/evolucion-comparada-de-la-pobreza-infantil-juvenil-y-de-los-mayores-en-euro-1, acceso 9 de julio de 2019

Finch, J. (1989). Family obligations and social change. Cambridge: Polity.

Foucault, M. (2002). ¿Qué es la ilustración? (2ª conferencia). En S. Mattoni (Ed.), ¿Qué es la ilustración? (pp. 81-108). Córdoba: Alcián.

Foucault, M. (2003). Hay que defender la sociedad. Curso del Collège de France (1975-1976). Tres Cantos; Madrid: Akal.

Foucault, M. (2008). Seguridad, territorio, población. Curso del Collège de France (1977-1978). Tres Cantos Madrid: Akal.

Gaitán Muñoz, L. (2018). Los derechos humanos de los niños: ciudadanía más allá de las "3Ps". Sociedad e infancias, 2, 17-37 (en línea). https://revistas.ucm.es/index.php/SOCI/article/view/59491, acceso 12 de julio de 2019.

Ginés, X. (2019). Ejemplos de innovación urbana: los huertos urbanos en Valencia. Conferencia presentado en Curso Ciudades en crisis y nuevas políticas urbanas, Universidad Autónoma de Barcelona (en línea). https://es.coursera.org/lecture/ciudades-politicas-urbanas/ejemplos-de-innovacion-urbana-los-huertos-urbanos-en-valencia-pye0X, acceso 12 de julio de 2019.

Hirikilab. (2015). Tecnologías blandas. Enredando con tecnologías sociales, cacharreando con las duras, rozándonos con las blandas (en línea). https://www.tabakalera.eu/es/tecnologias-blandas-0, acceso 12 de junio de 2019

Houtart, F. (2015). El carácter global de la agricultura campesina. En F. Hidalgo F, P. Lizárraga A., F. Houtart (Eds.), Agriculturas campesinas en América Latina. Propuestas y desafios (pp. 11-16). 
James, A. (2009). Agency. En J. Qvortrup, W. A. Corsaro, M.-S. Honig (Eds.), The Palgrave handbook of childhood studies (pp. 34-45). Basingstoke; New York: Palgrave Macmillan.

Jenson, J. (2006). The LEGOtm paradigm and new social risks: consequences for children. En J. Lewis (Ed.), Children, changing families and welfare states (pp. 27-50). Northampton, Mass.: Edward Elgar Pub.

Jin, Z. (2011). Global Technological Change: From Hard Technology to Soft Technology (2. ${ }^{a}$ ed.; K. W. Willoughby \& Y. Bai, Trads.). Bristol; Chicago: Intellect.

Leadbeater, C. (1997). The rise of the social entrepreneur. London: Nesta.

Lessig, L. (2006). Code and Other Laws of Cyberspace (Version 2.0). NY: Basic Books.

Lupetti, M. L. (2017). Shybo. An open-source low-anthropomorphic robot for children. HardwareX, 2(C), 50-50-60. 10.1016/j. ohx.2017.08.003

Marazzi, C. (2003). El sitio de los calcetines: el giro lingüístico de la economía y sus efectos sobre la política. Madrid: Akal.

Martínez Moreno, R., Cruz Gallach, H., Blanco, I., Salazar, Y. (2019). La innovación social ¿prácticas para producir autonomía, empoderamiento y nueva institucionalidad? Revista Internacional de Sociología, 77(2). 10.3989/ris.2019.77.2.17.022

Masschelein, J., y Simons, M. (2005). The strategy of the inclusive education apparatus. Studies in Philosophy and Education, 24(2), 117-138.

Mayall, B. (2009). Generational relations at family level. En J. Qvortrup, W. A. Corsaro, \& M.-S. Honig (Eds.), The Palgrave handbook of childhood studies (pp. 175-187). Basingstoke; New York: Palgrave Macmillan.

Millar, J. (2009). Understanding Social Security: Issues for Policy and Practice. Bristol: Policy Press.

Moreno Valle, P. C. (2018). Designing with our neighbors. Economía Creativa, 9, 52-83 (en línea). https://dialnet.unirioja.es/ servlet/articulo?codigo $=6799378$, acceso 12 de julio de 2019.

Moulaert, F., Martinelli, F., Swyngedouw, E., González, S. (eds.) (2010). Can neighbourhoods save the city? Community Development and Social Innovation. London: Routledge.

Moulier-Boutang, Y. (2011). Cognitive Capitalism. Oxford: Polity Press.

Mumford, L. (2009). Técnica y civilización. Extracto. En D. Mundo (Ed.), Lewis Mumford. Textos escogidos (pp. 21-101). Buenos Aires: Ediciones Godot.

Osborne, D., Gaebler, T. (1993). Reinventing government how the entrepreneurial spirit is transforming the public sector. New York: Plume.

Palley, E., Shdaimah, C. (2011). Child care policy: A need for greater advocacy. Children and Youth Services Review, 33(7), $1159-1165$.

Parziale, F., Scotti, I. (2016). Education as a Resource of Social Innovation. Sage Open, 6(3), 1-9. 10.1177/2158244016662691

Pearce, J. M. (2014). Open-Source Lab. How to Build Your Own Hardware and Reduce Research Costs. Elsevier.

Quesada Felice, M. Á., Matas, A. (2018). El huerto urbano como herramienta de transición socio-ambiental en la ciudad. Paradigma: revista universitaria de cultura, 21, 4-11 (en línea). https://dialnet.unirioja.es/servlet/articulo?codigo=6365836, acceso 12 de julio de 2019.

Qvortrup, J. (2007). European childhood - diverging or converging? En H. Wintersberger, L. Alanen, T. Olk, J. Qvortrup (Eds.), Childhood, generational order and the welfare state: exploring children's social and economic welfare (pp. 253-274). Odense: University Press of Southern Denmark.

Richter, F., Cuenca, J. (2018). Huertos de ocio y vida comunitaria. La agricultura urbana como experiencia de participación ciudadana. En A. Maradriaga Ortuzar, A. Ponce de León Elizondo (Eds.), Ocio y participación social en entornos comunitarios (pp. 189-212) (en línea). https://dialnet.unirioja.es/servlet/articulo?codigo=6817375, acceso el 12 de julio de 2019.

Runciman, B. (2017). The Kids Are Right (Aren't They). ITNOW, 59, 34.

Schor, J. B. (2006). Nacidos para comprar: los nuevos consumidores infantiles. Barcelona: Paidós.

Stallman, R. M. (2002). What is free software. En Free Society: Selected Essays of Richard Stallman (en línea). http://xill.irwinlaw.com/sites/default/files/attached/KP21\%2001\%20Stallman.pdf, acceso 12 de julio de 2019

Stallman, R. M. (2004). Software libre para una sociedad libre. Madrid: Traficantes de Sueños.

Ungerson, C. (1998). The informal sector. En P. Alcock, A. Erskine, M. May (Eds.), The Student's companion to social policy (Vol. 2, pp. 169-174). Oxford: Blackwell : Social Policy Association.

Unicef. (2017). El estado mundial de la infancia 2017. Niños en un mundo digital (en línea). https://www.unicef.es/sites/unicef. es/files/comunicacion/estado-mundial-infancia-2017.pdf, acceso 12 de julio de 2019.

Vesper, K. H. (1990). New Venture Strategies. Michigan: Prentice-Hall.

Vila-Viñas, D. (2012). El gobierno de la infancia: Análisis socio-jurídico del control y de las políticas de infancia contemporáneas, tesis doctoral. Universidad de Zaragoza (en línea). http://zaguan.unizar.es/record/9585/files/TESIS-2012-098.pdf, acceso 12 de julio de 2019.

Vila-Viñas, D. (2014a). El derecho a la vida familiar como derecho social. Afecciones de la crisis sobre los estilos de vida familiar y posibilidades de protección jurídica. En M. Calvo García, M. J. Bernuz Beneitez (eds.), Derechos económicos, sociales y culturales (pp. 285-310), Valencia: Tirant lo Blanch.

Vila-Viñas, D. (2014b). La gobernabilidad más allá de Foucault. Un marco para la teoría social y política contemporáneas. Zaragoza: Prensas Universitarias de Zaragoza.

Vila-Viñas, D., Araya, D., Bouchard, P. (2015). Educación: Recursos educativos abiertos. En D. Vila-Viñas y X. E. Barandiaran (eds.), Buen Conocer-FLOK Society. Modelos sostenibles y políticas públicas para una economía social del conocimiento 
común y abierto en el Ecuador (pp. 61-142). Quito: IAEN - CIESPAL (en línea). http://book.floksociety.org/ec/1/1-1-educacion-recursos-educativos-abiertos, acceso 12 de julio de 2019.

Walkerdine, V. (1995). Psicología del desarrollo y pedagogía centada en el niño: la inserción de Piaget en la educación temprana. En J. Larrosa Bondía (Ed.), Escuela, poder y subjetivación (pp. 77-152). Madrid: Ediciones de La Piqueta.

Wintersberger, H. (1994). Los niños y la sociedad. En Consejo de Europa (Ed.), Políticas de infancia (pp. 53-89). Madrid: Ministerio de Asuntos Sociales.

Wintersberger, H., Olk, T. (2007). Welfare state and generational order. En H. Wintersberger, L. Alanen, T. Olk, J. Qvortrup (Eds.), Childhood, generational order and the welfare state: exploring children's social and economic welfare (pp. 59-90). Odense: University Press of Southern Denmark.

Xie, X., Huang, C.-C., Chen, Y., Hao, F. (2019). Intelligent robots and rural children. Children and Youth Services Review, 100, 283-290.

Zuil, M. (2019, mayo 6). Adiós al fútbol en el patio: los colegios se reinventan para que todos jueguen. El Confidencial (en línea). https://www.elconfidencial.com/alma-corazon-vida/educacion/2018-10-20/patios-inclusivos-futbol-adios_1633102/, acceso 12 de julio de 2019. 